\title{
Effectiveness of Qualitative Research Methods: Interviews and Diaries
}

\author{
Wafaa Abdullah Alamri \\ Correspondence: Wafaa Abdullah Alamri, Lecturer at English Language Institute, University of Jeddah, Saudi Arabia.
}

Received: November 1, 2018

doi:10.11114/ijecs.v2i1.4302

\author{
Accepted: January 11, 2019 Online Published: May 28, 2019
}

URL: https://doi.org/10.11114/ijecs.v2i1.4302

\begin{abstract}
The present study aims to explore the effectiveness of qualitative research methods. The qualitative research method has been opted after a thorough literature examination. The concept of triangulation and the process of multi-method qualitative research on error correction and students' motivation were also examined. The results discovered more about the participants as he or she reads their diaries containing their detailed opinions and feelings. Subjectivity is another remarkable feature of diaries for learners to improve their writing skills and to use diverse vocabulary to express their views and emotions. Reviewing the information within a diary helps people to judge their behaviour and others, towards certain events. It was depicted that the triangulation method assists to have a more in-depth understanding of the recorded entries in the diaries by using semi-structured interviews. Both approaches were found to comprise of advantages and disadvantage, where the selection is based on the nature of the study and the understanding of the researcher. The results of the present study help guide the researcher in determining the specific choice of study.
\end{abstract}

Keywords: interviews, semi-structured interviews, diary study, triangulation, multi-method study

\section{Introduction}

A wide range of techniques and methods are used to examine people's experiences, beliefs, and feelings by employing qualitative research design. Qualitative research emphasized on the linguistic data that identify issues from the study participants' perspectives considering a particular area. The researcher is required to understand the meanings and interpretations of the responses, obtained through the participants of the study regarding their behavior, events, and objects (Hennink et al., 2011; Bowling, 2014). Silverman (2016) has mentioned that qualitative research plays a significant role in settings to obtain open-ended data. The qualitative design is highly substantial for assessing thoughts, views, and perspectives to present information collectively. Merriam \& Tisdell (2015) have also shown that qualitative design is significantly helpful for the researchers to collect a broader range of data from a diverse population. Practically in the larger populations, thoughts and perspectives are judged through interviews, which is a conventional technique under qualitative research method (Silverman, 2013; Bryman \& Bell, 2015).

Qualitative research methods including interviews, observations, diaries, and focus groups are commonly used to conduct academic and psychological research. The present study compared two qualitative research methods (interviews and diaries) and highlighted differences between the two techniques. The similarities and variations have been considered from the perspectives of both the researcher and the participants. Also, the concept of triangulation and the process of multi-method qualitative research on error correction and students' motivation have been examined.

\subsection{Interviews as a Data Collection Technique}

The interview as a method of qualitative data collection involves two people discussing a specific human or social issue. Using interview techniques, the researcher commonly aims to obtain the perspective of the interviewee by interpreting the meaning of the described phenomena (Kvale, 1996, p. 6). This flexible technique allows the interviewer and interviewee to elaborate on the topic to pursue an idea or response in greater detail.

\subsection{Types of Interviews}

Interviews can be structured, semi-structured, and unstructured. The structured interview format compels the researcher to use precisely the same questions with all participants, while the semi-structured interview format allows the researcher to add or omit any aspect of the pre-planned questions. The semi-structured interview consists of several key questions that help to define the areas that are being explored in the research. The researcher tends to use semi-structured interviews to enable the interviewee to elaborate on certain issues (Dörnyei, 2007, p. 136). Conversely, unstructured interviews require no question guide. Generally, interviewing is considered as the most popular qualitative 
method, used by researchers. Moreover, interviews can also be readily combined with other approaches in a multi-method design (Brewerton \& Millward, 2001, p. 69). Some researchers combine interviews with another research method to test and verify the truthfulness of the collected data.

\subsection{Advantages and Disadvantages of Qualitative Tools}

As a qualitative tool, interviews have obvious advantages and disadvantages, in which the main advantage is related to the issue of flexibility. The researcher can adjust and change the direction of the questions, especially in a semi-structured interview. The flexible nature of interviews also helps the participants to reveal more about themselves, which, in turn, serves to enrich the qualitative data. This effective instrument allows the interviewer to effectively explore the interviewee's thoughts, feelings, and opinions. The interviewer can follow up on the thoughts, feelings, and ideas behind the responses in a way that other methods do not allow. The subjective nature of interviewing is reflected as the interviewee expresses through the opinions and experiences. This nature helps the interviewer to know more about the interviewee. In addition, interviewing provides an opportunity to explain or clarify questions or answers, thereby helping to increase the accuracy of the collected data. The interviewer, who is often a researcher, can explain the questions to the interviewee, who then has the opportunity to clarify and elaborate answers. The personal interaction that takes place between the two parties facilitates the development of the relationship between the researcher and the participants. According to King and Horrocks (2010), "Development of relationship is widely seen as a key ingredient in successful qualitative interviewing" ( $($. 48). The interaction that occurs during the interview process supplies the data as well as any relevant additional information. Another valuable advantage of the qualitative interview is that it can be taped or video-recorded, which helps with the transcription, coding, and analysis of the data. However, there are also disadvantages, which include the fact that conducting interviews is time-consuming, primarily if they are recorded and then fully transcribed. The researcher needs time to arrange the interview schedule, collect and record the data, transcribe code, and analyze the data (Bryman, 2012, p. 228). Scheduling appropriate time is a prominent interview weakness, which may affect the respondents' answers.

Sometimes respondents are more concerned about the social norms of the research topic than expressing their personal opinions or feelings about it. The participants' responses and the collected data are not considered trustworthy, which, in turn, affects the study results. Diary study is a longitudinal method of gathering qualitative data from participants as they undergo certain experiences, and it is a useful way to access personal detailed meanings and understandings. The researcher asks the participants to record their opinions, thoughts or feelings about a specific issue. Diaries are also preferable when the investigation is oriented towards the social sciences. For instance, some researchers use diaries to study psychological aspects, such as motivation and perception. Thus, diaries provide direct "access to information that is otherwise hard to obtain" (Willing, 2013, p. 34).

\subsection{Triangulation Method}

Triangulation is defined by Flick (2009) as "the combination of different methods, study groups, local and temporal settings, and different theoretical perspectives in dealing with a phenomenon" (p. 444). When conducting a study, the researcher may need to use two or more methods to investigate a specific topic. By combining different research methods or instruments, the researcher checks the results to increase the credibility and validity of the data. Triangulation, therefore, validates the research data by verifying the information.

\section{Method}

The qualitative research approach has been opted to explore and understand the methods of the qualitative approach. A total of 13 published studies have been investigated to understand the effectiveness of qualitative methods based on interviews and relevant techniques used. Interviews and diary studies have been selected, which are considered as the most popular qualitative research methods. Scholarly searches assisted in investigating the effectiveness of the qualitative approach. The study examined the pros and cons of using a diary as a qualitative data collection instrument, which helps in collecting longitudinal information. The qualitative approach has been adopted by many successful types of research, which have been analyzed through the literature examination (Smith, 2015; Silverman, 2016; Taylor et al., 2015).

To contrast diaries and semi-structured interviews, the study initially examined some points, which highlighted the primary differences between the two instruments. Data collection via interview involves an interviewer, typically the researcher, who asks pre-planned questions from an interviewee. The interviewer follows the question guide as he or she interviews the participants. Through the interviewer's questions and comments, the relationship is established.

To compare diaries and interviews, the study has examined the similarities from the perspectives of the researcher and the participant. Diaries and interviewing as qualitative methods are characterized by the subjectivity of participants as they portray themselves in the recorded data. The study was only limited to the qualitative research method. However, 
there are also other methods based on quantitative research methods (Creswell, 2013), which can also be explored in future researches.

\section{Results and Discussion}

It is essential to examine the pros and cons of using a diary as a qualitative data collection instrument. Collecting longitudinal information is considered the primary objective of the diary study. The participants may be required to recall events that they experienced four or five months ago to support the research data. This longitudinal information helps the researcher to discover more about the participants as he or she reads their diaries containing their detailed opinions and feelings. Reviewing the information within a diary assists people to judge their own behavior, or that of others, towards certain events. Furthermore, diaries are characterized by introspection, as they deal with the participants' insider meanings; thereby, allowing the researcher to read their thoughts and emotions related to a specific context.

Subjectivity is another remarkable feature of diaries. Researchers want to capture the experiences as participants live or see them. For the purpose of conducting educational research, teachers and learners might be asked to write in diaries after their classes. They can explain situations using their own comments and thoughts. A diary is the most familiar way to record and respond to events, and this familiarity is recognized as an advantage of conducting a diary study. It also helps the learners to improve their writing skills and to use diverse vocabulary to express their opinions and emotions.

Despite the positive features of diaries as a qualitative research instrument, there are also considerable weaknesses. Diaries are self-recorded documents; therefore, there is a perceived source of biases within this method. Social desirability biases invalidate diary study results because respondents may not provide accurate or complete information concerning the research topic because the answers were recorded that correspond to what is socially desirable. Sometimes, participants are asked to record experiences that occurred long ago, and they might not remember the information or may remember it inaccurately, whether consciously or not; or they might self-select materials depending on their desire. This method of recalling information affects the accuracy of the data. In terms of the reliability of responses, diary entries need to be verified using another research method, as suggested by Fife-Schaw (1995), who stated that the researcher could "use interview techniques to act as a verification check for diary entries" (p. 112). Diaries are also time-consuming in terms of data collection and analysis. The researcher needs to dedicate a substantial amount of time to arrange training sessions on how to maintain diaries, collecting, coding, and analyzing the data. Unlike numerous other research methods, diary studies are not available for researchers to consult writing in diaries, which are considered as the most familiar way to record experiences and events. Table 1 has illustrated the strengths and weaknesses of using diaries.

Table 1. Strengths and weaknesses of diaries

\begin{tabular}{ll}
\hline Strengths & Weaknesses \\
\hline Capability to judge one's behavior towards certain events & Self-recorded documents \\
Ability to deal with participant's insider meaning & May have perceived sources of biases \\
Subjectivity & Social desirability biases \\
Capturing of the experiences & A concern of accurate and complete information \\
Recognition of familiarity & Time-consuming in terms of data collection \\
Use of vocabulary to express opinions and emotions & Time-consuming in terms of data analysis \\
\hline
\end{tabular}

The subjectivity in diaries is more obvious, as the participants use this self-recording instrument to make in-depth comments on specific problems or issues. While conducting interviews, the researcher takes part in the interview him or herself, asks pre-planned questions and clarifies them for the participants if needed; however, the presence of the researcher can have a considerable effect on the participant's behavior and answers. Hitchcock and Hughes (1989) argued that the researcher inevitably has some influence on the interview and, thereby, on the data (Cohen et al., 2013, p. 204). Whereas in diary studies, participants go off and create data themselves, and the researcher only becomes involved in the analytical stage (Giles, 2013, p. 262).

When conducting diary studies or interviews, social desirability bias is a crucial factor. As interviews require the intervention of the researcher, the participants provide more biased and less reliable data, whereas participants in diary studies tend to produce more accurate and less biased data; this is because they are using a self-report instrument to capture experiences close to the time of their occurrence. In terms of data analysis, researchers tend to analyze the diary and interview data in the same way. After collecting the data, the researcher reads the diaries; starts coding the entries into meaningful categories, which helps to organize large amounts of text; and conducts coding to capture how respondents define a particular aspect of an issue. The researcher then groups similar ideas to make it easier to search 
for them. Likewise, the researcher needs to transcribe the interview data which have been video/audio recorded before the researcher codes them. For both diary studies and interviews, the data analysis is iterative; the researcher tends to return frequently to the collected data to ensure the accuracy of information and meaning. During the interview process, the relationship between the researcher and the participant allows the former to achieve iteration; while, analyzing the data. Similarly, the researcher may revisit the collected diary data to ensure the accuracy of information and meaning. Thus, as qualitative data collection tools, interviews and diaries share some similarities (Table 2).

Table 2. Strengths and weaknesses of Interview

\begin{tabular}{ll}
\hline Strengths & Weaknesses \\
\hline Practices controlled data collection procedure. & $\begin{array}{l}\text { Interviewer presence affects the participant's behavior and } \\
\text { answers. }\end{array}$
\end{tabular}

Provides more comprehensive understanding through Intervention promotes biases among the participants. triangulation.

Enables the researcher to introspect and reflect on the Less reliable data. collected data.

The preplanning of the interviews helps the interviewer to Time-consuming in terms of data analysis. guide the session.

Promotes the researcher to clarify any misgivings related Time-consuming in terms of data transcribing. an answer.

Better iteration by interaction among the participants and Recalling the previous events or situations. interviewer.

The interviewer directs the interview to obtain the data that will answer the research questions. Conversely, when using the diary study method, the researcher provides the participants with guidance before beginning to record, and this is done without establishing any kind of rapport between them. The interviewer simply asks the participant to maintain a diary on a certain issue or problem, and then he or she leaves the participant to do so. Unlike the controlled data collection of an interview, diary entries are difficult for the researcher to control in terms of content, length, and depth, as participants write their thoughts and comments concerning specific problems or issues. According to Breakwell and Wood (1995), "Participants will invariably misremember information, either consciously or not, and will also self-select material depending on their motivation" (Brewerton \& Millward, 2001, p. 112).

The triangulation method assists to have a more in-depth understanding of the recorded entries in the diaries by using semi-structured interviewing. It would also provide an opportunity for the introspection and reflection which could be missing from the written diaries. In addition, the diary and interview method would help to check the validity and credibility of the findings.

After collecting the diary entries, the coding and grouping of the entries would be a crucial part of the process. Relevant ideas, techniques, opinions, activities and/or any related concepts would be coded. The data can be further grouped according to the important ideas, such as the research techniques used to correct errors, students' favorites techniques, and their opinions on these techniques. Coding and grouping effectively facilitate data analysis. While coding, some points would be highlighted, such as the relevant techniques used to correct errors, opinions, and phrases, as well as any surprising or important materials. The coded data would then be categorized according to their importance to the study, and the unimportant entries would be removed. The most commonly used, preferred, and disliked techniques would be coded for them to be discussed and elaborated in detail during the interviews.

Concerning semi-structured interviews, open-ended vital questions can be developed to ask the participants after analyzing the data from their diaries. These pre-planned questions would provide guidance, as the interviewer would discuss the interviewees regarding the main topics. Semi-structured interviews allow great clarification and understanding to take place. The key questions of the interview can be related primarily to error correction techniques and, more specifically to the methods that participants prefer and find most helpful. These questions would then be elaborated during the interview process using follow-up questions. The participants can be able to justify their diary entries in their answers.

It is important to mention that all the interviews would be audio-recorded (Doody \& Noonan, 2013). After collecting the interview data, the transcription process can be performed before reading the transcripts carefully one by one. Coding of the transcribed data can be started by labeling the relevant words, techniques, sentences, opinions and whatever else that is relevant. The most important codes can be identified and categorize them by combining the relevant ones, and several codes might be created while re-reading the transcripts. After coding, the codes can be grouped according to the 
relationship between them and then omit the unnecessary ones. The grouped codes might be techniques used in error correction, students' opinions or preferred techniques.

Table 3 presents the relative comparison between interviews and diary method. Based on the analysis, it is found that the reliability and authenticity of data are prevalent in both methods. However, the degree of reliability and authenticity may vary for both the methods such as ADM (2011) has argued that interview requires the participant to recall a certain event after some time, which may influence the information provided. Whereas, in the diary method, the recorded information includes information in a real scenario, new to the event, which demonstrated more true depiction. Reflecting upon the depth and flexibility of information, interview method is found dominant. Similar findings have also been pinpointed by Dikko (2016), who has indicated that the intervention of the researcher or the interviewer in adopting it enables clarifying the provided information which is found lacking in the dairy method.

George et al (2016) have also asserted that flexibility is absent in the diary method as a result of instructions, which may pose challenges in understating, making its careful planning pertinent. The confidentiality and anonymity are evident in the diary method as the participants are not in direct contact with each, where direct involvement with the interviewer impacts their confidentially and anonymity. These findings are in line with the research of Anttila et al. (2017), in which the electronic dairy method was used. Biases prevalence is reported in the interview as the respondent may alter his answers based upon the intervention by the interview which may have been differ in his absence. Sivell et al. (2019) have also highlighted that no intervention serves as an advantage in a direct method as respondents are able to write their exact thoughts and feeling without being judged. Lastly, time is significantly consumed in both the methods given their similar procedure of analysis, though, interview give additional emphasis to transcribe the audio-taped discussion with the participant. This is also supported by ADM (2011). However, despite the differences among the two methods, several studies have promoted the researcher to assimilate both the methods for drawing a holistic picture of the actual research problem (Thomas et al., 2015; Janssens, 2018).

Table 3. Comparison of Diary and Interview Method

\begin{tabular}{lll}
\hline Difference & Diary Method & Interview Method \\
\hline Reliable Data & $\checkmark$ & $\checkmark$ \\
Accurate Data & $\checkmark$ & $\checkmark$ \\
Flexibility & & \\
Comprehensive Data & $\checkmark$ & \\
Confidentiality & $\checkmark$ & $\checkmark$ \\
Anonymity & & \\
Bias & $\checkmark$ & \\
Time Consumption & & \\
\hline
\end{tabular}

\section{Conclusion}

The study has effectively contributed to examine the diaries and interviews by comparing and contrasting them as qualitative data collection instruments. The triangulation method was also discussed and focused on examining the techniques, used to correct the errors and analyze their levels of motivation. The study examined the pros and cons of using a diary as a qualitative data collection instrument. Collecting longitudinal information is considered the primary objective of the diary study. Participants may be required to recall events that they experienced four or five months ago to support the research data, which would help in discovering more about the participants as he or she reads their diaries containing their detailed opinions and feelings. The study results have combined the results obtained from diaries and the interviews to describe them and note how they are connected. It has been shown that direct quotes from the diaries and interviews were useful. Moreover, future studies should consider the connections between groups because they are the main outcomes.

\section{Acknowledgements}

The author is very thankful to all the associated personnel in any reference that contributed in/for the purpose of this research. The author declares no competing interest.

\section{References}

ADM, S. W. P. M. P. (2011). The combined use of diaries and interviewing for the collection of data in midwifery research. Evidence Based Midwifery, 66.

Anttila, K. I., Anttila, M. J., Kurki, M. H., \& Välimäki, M. A. (2017). Social relationships among adolescents as 
described in an electronic diary: a mixed methods study. Patient preference and adherence, $11,343$. https://doi.org/10.2147/PPA.S126327

Bowling, A. (2014). Research methods in health: investigating health and health services. McGraw-Hill Education (UK).

Brewerton, P., \& Millward L. (2001). Organizational research methods: A guide for students and researchers. London: SAGE. https://doi.org/10.4135/9781849209533

Bryman, A. (2012). Social research methods. Oxford University Press.

Bryman, A., \& Bell, E. (2015). Business research methods. Oxford University Press, USA.

Cohen, L., Manion, L., \& Morrison, K. (2013). Research methods in education (7th ed.). London: Routledge. https://doi.org/10.4324/9780203720967

Creswell, J. W. (2013). Research design: Qualitative, quantitative, and mixed methods approaches. Sage publications.

Dikko, M. (2016). Establishing construct validity and reliability: Pilot testing of a qualitative interview for research in Takaful (Islamic insurance). The Qualitative Report, 21(3), 521-528.

Doody, O., \& Noonan, M. (2013). Preparing and conducting interviews to collect data. Nurse researcher, 20(5), 28-32. https://doi.org/10.7748/nr2013.05.20.5.28.e327

Dörnyei, Z. (2007). Research methods in applied linguistics: Quantitative, qualitative, and mixed methodologies. Oxford: Oxford University Press.

Fife-Schaw, C. (1995). Questionnaire design. Research methods in psychology, 2, 158-74.

Flick, U. (2009). An introduction to qualitative research. Los Angeles: Sage Publications.

George, G., Osinga, E. C., Lavie, D., \& Scott, B. A. (2016). Big data and data science methods for management research. https://doi.org/10.5465/amj.2016.4005

Giles, D. (2013). Advanced research methods in psychology. Routledge. https://doi.org/10.4324/9780203759851

Hennink, M. M., Hutter, I., \& Bailey, A. (2011). Qualitative research methods. London: SAGE.

Hitchcock, G., \& Hughes, D. (1995). Research and the teacher: A qualitative introduction to school-based research. Psychology Press.

Janssens, K. A., Bos, E. H., Rosmalen, J. G., Wichers, M. C., \& Riese, H. (2018). A qualitative approach to guide choices for designing a diary study. BMC medical research methodology, 18(1), 140. https://doi.org/10.1186/s12874-018-0579-6

King, N., \& Horrocks C. (2010). Interviews in qualitative research. Thousand Oaks, CA: SAGE.

Kvale, S. (1996). InterViews: An introduction to qualitative research interviewing.

Merriam, S. B., \& Tisdell, E. J. (2015). Qualitative research: A guide to design and implementation. John Wiley \& Sons.

Silverman, D. (2013). Doing qualitative research: A practical handbook. SAGE Publications Limited.

Silverman, D. (Ed.). (2016). Qualitative research. Sage.

Sivell et al. (2019). Considerations and recommendations for conducting qualitative research interviews with palliative and end-of-life care patients in the home setting: a consensus paper. BMJ supportive \& palliative care, 9(1), e14-e14. https://doi.org/10.1136/bmjspcare-2015-000892

Smith, J. A. (Ed.). (2015). Qualitative psychology: A practical guide to research methods. Sage.

Taylor, S. J., Bogdan, R., \& DeVault, M. (2015). Introduction to qualitative research methods: A guidebook and resource. John Wiley \& Sons. Thousand Oaks, CA: Sage Publications.

Thomas et al. (2015). Comparison of fieldworker interview and a pictorial diary method for recording morbidity of infants in semi-urban slums. BMC public health, 15(1), 43. https://doi.org/10.1186/s12889-015-1372-7

Willing, C. (2013). Introducing qualitative research in psychology. Berkshire: Open University Press.

\section{Copyrights}

Copyright for this article is retained by the author(s), with first publication rights granted to the journal.

This is an open-access article distributed under the terms and conditions of the Creative Commons Attribution license (http://creativecommons.org/licenses/by/4.0/). 